\title{
A NOTE ON NON-AUTONOMOUS IMPLICIT INTEGRAL EQUATIONS WITH DISCONTINUOUS RIGHT-HAND SIDE
}

\author{
GIOVANNI ANELLO AND PAOLO CUBIOTTI
}

ABSTRACT. Let $I:=[0,1], f: I \times[0, \sigma] \rightarrow \mathbf{R}, g: I \times I \rightarrow$ $[0,+\infty[$ and $h: I \times] 0,+\infty[\rightarrow \mathbf{R}$. In this note we prove an existence result for solutions $u \in L^{s}(I)$ of the integral equation

$$
h(t, u(t))=f\left(t, \int_{I} g(t, z) u(z) d z\right) \text { for a.a. } t \in I
$$

where, in particular, the continuity of $f$ with respect to the second variable is not assumed. Our result is a partial extension of a previous result of the same authors [1], where the function $h$ was not allowed to depend explicitly on $t$.

1. Introduction. Let $A \subseteq] 0,+\infty[, I:=[0,1]$ and $J:=[0, \lambda]$, with $\lambda>0$, and let us consider the integral equation

$$
h(u(t))=f\left(t, \int_{I} g(t, z) u(z) d z\right) \text { for a.a. } t \in I,
$$

where $f: I \times J \rightarrow \mathbf{R}, g: I \times I \rightarrow[0,+\infty[$ and $h: A \rightarrow \mathbf{R}$. Recently, in the papers $[\mathbf{1}, \mathbf{2}, \mathbf{3}, \mathbf{6}]$, the equation (1) has been investigated together with some of its special cases, obtaining some existence results where the function $f$ is not assumed to be continuous in the second variable. More specifically, in the paper [1] the function $f$ was assumed to satisfy the following assumption: there exist a function $f^{*}: I \times J \rightarrow \mathbf{R}$ and two negligible sets $E_{1}, E_{2} \subseteq J$, with $E_{2}$ closed, such that $f^{*}(\cdot, x)$ is measurable for each $x$ in a countable dense subset of $J$ and, for a.a. $t \in I$, one has

$$
\left\{x \in J: f^{*}(t, x) \neq f(t, x)\right\} \subseteq E_{1},
$$

$\left\{x \in J: f^{*}(t, \cdot)\right.$ is discontinuous at $\left.x\right\} \subseteq E_{2}$.

\footnotetext{
2000 AMS Mathematics subject classification. Primary 45P05, 45G10, $47 \mathrm{G} 10$.

Keywords and phrases. Implicit integral equations, discontinuity, lower semicontinuous multi-functions, operator inclusions, selections.

Received by the editors on February 23, 2006, and in revised form on June 2, 2006.

Copyright (C)2007 Rocky Mountain Mathematics Consortium
} 
Of course, such a function $f$ can be discontinuous (with respect to the second variable) at each point $x \in J$.

In this note we consider the more general integral equation

$$
h(t, u(t))=f\left(t, \int_{I} g(t, z) u(z) d z\right) \quad \text { for a.a. } t \in I,
$$

and, in the spirit of [1] , we prove an existence theorem for solutions $u \in L^{s}(I)$ where $f$ is not assumed to satisfy the usual Carathéodory condition, but only the more general condition above. Our study was motivated by the reading of the papers $[\mathbf{4}, \mathbf{7}, \mathbf{9}]$. In particular, in [7], to find solutions to equation (2), besides assuming the Carathéodory condition on $f$ and $h$, it was assumed that $h$ is nondecreasing in $] 0,+\infty$ [ with respect to the second variable (and having linear growth). In $[4,9]$ the authors consider little different implicit integral equations. However, many special cases of these latter fall within the framework of equation (2). We also refer the reader to the references of these papers where he can find some mathematical models which make interesting studying equations like (2).

Our result is as follows (from now on, $m$ denotes the Lebesgue measure on the real line and "int" stands for "interior"; moreover, to make notations simpler, we put $\left.h_{t}:=h(t, \cdot)\right)$.

Theorem 1.1. Let $I:=[0,1]$ and $J:=[0, \lambda]$, with $\lambda>0$. Let $s \in] 1,+\infty], A \subseteq] 0,+\infty[$ an interval, $h: I \times A \rightarrow \mathbf{R}$ a Carathéodory function. Let $f: I \times J \rightarrow \mathbf{R}, g: I \times I \rightarrow\left[0,+\infty\left[, \beta \in L^{s}(I)\right.\right.$, $\phi_{0} \in L^{j}(I)$, with $j \geq s^{\prime}:=s /(s-1)$ and $j>1, \phi_{1} \in L^{s^{\prime}}(I)$, and let $P$ be a countable dense subset of $J$. Assume that:

(i) there exist a function $f^{*}: I \times J \rightarrow \mathbf{R}$ and two sets $E_{1}, E_{2} \subseteq J$, with $E_{2}$ closed and $m\left(E_{1} \cup E_{2}\right)=0$ such that, for each $x \in P$, the function $f^{*}(\cdot, x)$ is measurable and for a.a. $t \in I$ one has

$$
\left\{x \in J: f^{*}(t, x) \neq f(t, x)\right\} \subseteq E_{1}
$$

and

$$
\left\{x \in J: f^{*}(t, \cdot) \text { is discontinuous in } x\right\} \subseteq E_{2} \text {; }
$$


(ii) for a.a. $t \in I$, one has that

$$
\operatorname{int} h_{t}^{-1}(z)=\varnothing \text { for all } z \in \operatorname{int} h_{t}(A),
$$

and the two sets

$$
\begin{aligned}
& \left\{x \in A: x \text { is a local minimum for } h_{t}\right\}, \\
& \left\{x \in A: x \text { is a local maximum for } h_{t}\right\}
\end{aligned}
$$

are closed;

(iii) if one puts

$$
v(t):=\operatorname{essinf}_{x \in J} f(t, x), \quad z(t):=\operatorname{esssup}_{x \in J} f(t, x),
$$

then for a.a. $t \in I$ one has

$$
[v(t), z(t)] \subseteq h_{t}(A) \text { and } \sup h_{t}^{-1}([v(t), z(t)]) \leq \beta(t) ;
$$

(iv) one has

$$
0<\left\|\phi_{0}\right\|_{L^{s^{\prime}}(I)} \leq \frac{\lambda}{\|\beta\|_{L^{s}(I)}}
$$

(v) for each $t \in I$, the function $g(t, \cdot)$ is measurable;

(vi) for a.a. $z \in I$, the function $g(\cdot, z)$ is continuous in I, differentiable in $] 0,1[$ and

$$
\left.g(t, z) \leq \phi_{0}(z), \quad 0<\frac{\partial g}{\partial t}(t, z) \leq \phi_{1}(z) \quad \text { for all } t \in\right] 0,1[.
$$

Then there exists a solution $\hat{u} \in L^{s}(I)$ to the equation (2).

Theorem 1.1 above extends Theorem 1.1 of [1] (where equation (1) is considered) to the more general equation (2) (note also that assumptions (ii) $)_{1}$ and (iii) of Theorem 1.1 are a natural extension of assumptions (6) and (7) of Theorem 1.1 of [1] to the case in which $h$ depends explicitly on $t$ ). Such an extension is not full since we are assuming, in addition, that for a.a. $t \in I$ both of the sets of local minima 
and local maxima of $h_{t}$ are closed. Roughly speaking, this is needed in order to prove Lemma 2.4 below and, consequently, to ensure that the multi-function $G$ defined in the proof of Theorem 1.1 is $\mathcal{L}(I) \otimes \mathcal{B}(J)$ measurable. Ultimately, this is the key point of the proof, which makes it possible to extend the ideas of [1] to problem (2).

The proof of Theorem 1.1 will be given in the next section, together with some technical preliminary results. As regards notations, we refer to [1]. Finally, we refer to [1] for remarks and counterexamples to possible improvements of Theorem 1.1.

2. The proof of Theorem 1.1. Firstly, we establish some technical lemmas. We recall that if $(T, \Im)$ is a measurable space and $X$ is a topological space, a multi-function $F: T \rightarrow 2^{X}$ is said to be measurable if $F^{-}(A):=\{t \in T: F(t) \cap A \neq \varnothing\} \in \Im$ for every open set $A$ in $X$.

Lemma 2.1. Let $(T, \Im), X$ and $Y$ be respectively a measurable space, a separable metric space and a topological space. Let $h: T \times X \rightarrow Y$ be a function such that $h(t, \cdot)$ is continuous for all $t \in T$ and $h(\cdot, x)$ is measurable for all $x \in X$. Let $\Omega$ be a subset of $X$.

Then, the multi-function $H: T \rightarrow 2^{Y}$ defined by $H(t)=h(t, \Omega)$ for every $t \in T$, is measurable.

Proof. Since every subset of a separable metric space is separable with respect to the relative topology, we can find a sequence $\left\{x_{n}\right\}$ in $\Omega$ such that $\Omega \subseteq \overline{\left\{x_{n}\right\}}$. Now, let $A$ be an open subset of $Y$. We now show that

$$
H^{-}(A)=\bigcup_{n \in \mathbf{N}}\left\{t \in T: h\left(t, x_{n}\right) \in A\right\} ;
$$

hence, our conclusion will follow. Clearly, it is enough to prove that

$$
H^{-}(A) \subseteq \bigcup_{n \in \mathbf{N}}\left\{t \in T: h\left(t, x_{n}\right) \in A\right\}
$$

since the reverse inclusion is obvious. To this aim, choose any $t^{*} \in$ $H^{-}(A)$. Then, there exists $x \in \Omega$ such that $h\left(t^{*}, x\right) \in A$. Since the function $h\left(t^{*}, \cdot\right)$ is continuous at $x$, there exists $n^{*} \in \mathbf{N}$ such that $h\left(t^{*}, x_{n^{*}}\right) \in A$. Consequently, we get 


$$
t^{*} \in \bigcup_{n \in \mathbf{N}}\left\{t \in T: h\left(t, x_{n}\right) \in A\right\}
$$

as claimed.

In the sequel, if $T$ is a given nonempty set, $A$ is a topological space and $h: T \times A \rightarrow \mathbf{R}$ is a function, we put, for each $t \in T$,

$$
\begin{aligned}
m_{h}(t) & :=\left\{x \in A: x \text { is a local minimum for } h_{t}\right\} \\
M_{h}(t) & :=\left\{x \in A: x \text { is a local maximum for } h_{t}\right\} \\
l_{h}(t) & :=\inf _{x \in A} h_{t}(x) \\
u_{h}(t) & :=\sup _{x \in A} h_{t}(x) .
\end{aligned}
$$

Lemma 2.2. Let $T$ be a topological space with a $\sigma$-algebra $\mathcal{T}$ containing the open subsets of $T$. Let $A \subseteq \mathbf{R}$ be an interval, and let $h: T \times A \rightarrow \mathbf{R}$ be a continuous function satisfying

$$
\operatorname{int} h_{t}^{-1}(r)=\varnothing \text { for all } t \in T, r \in\left[l_{h}(t), u_{h}(t)\right] .
$$

Then, the multi-functions $m_{h}, M_{h}: T \rightarrow 2^{A}$ are measurable.

Proof. Put

$$
\begin{aligned}
& T_{1}:=\left\{t \in T: l_{h}(t)>-\infty\right\}, \\
& T_{2}:=\left\{t \in T: u_{h}(t)<+\infty\right\} .
\end{aligned}
$$

It is easy to realize that $T_{1}$ and $T_{2}$ are measurable, see [5, Lemma III.39]. Now, we observe that, for each $t \in T$, one has

$$
m_{h}(t)=m_{1}(t) \cup m_{2}(t) \cup m_{3}(t)
$$

where

$$
\begin{aligned}
& m_{1}(t):=\left\{x \in A: h(t, x)=l_{h}(t)\right\} \\
& m_{2}(t):=\left\{x \in m_{h}(t): h(t, x)=u_{h}(t)\right\} \text { and } \\
& m_{3}(t):=\left\{x \in m_{h}(t): h(t, x) \in\right] l_{h}(t), u_{h}(t)[\} .
\end{aligned}
$$


The continuity of $h$ implies that $m_{1}$ has closed values in $A$. Moreover, the functions $l_{h}$ and $u_{h}$, which take their values in $\overline{\mathbf{R}}$, are upper semicontinuous and lower semi-continuous in $T$, respectively. In particular, they are measurable. Consequently, the functions

$$
(t, x) \in T_{1} \times A \longrightarrow h(t, x)-l_{h}(t) \in \mathbf{R}
$$

and

$$
(t, x) \in T_{2} \times A \longrightarrow h(t, x)-u_{h}(t) \in \mathbf{R}
$$

have the Carathèodory property. Then, by [8, Theorem 3.5 and Corollary 6.3], it follows that $m_{1}$ is measurable.

Now, we claim that $m_{2}$ is measurable too. To prove our claim, fix an open subset $\Omega$ of $A$. Moreover, let $r_{n}$ be a sequence in $\Omega$ such that $\Omega \subseteq \overline{\left\{r_{n}\right\}}$, and put

$$
B_{n, k}:=\left\{y \in A:\left|y-r_{n}\right|<1 / k\right\} .
$$

Of course, each $B_{n, k}$ is open in $A$. Let us show that

$$
m_{2}^{-}(\Omega)=\bigcup_{(n, k) \in \mathbf{N} \times \mathbf{N}}\left\{t \in T_{2}:\{0\}=h\left(t, B_{n, k} \cap \Omega\right)-u_{h}(t)\right\}
$$

by which, taking into account Lemma 2.1, our claim follows. Let $t \in m_{2}^{-}(\Omega)$. Then $m_{2}(t) \cap \Omega \neq \varnothing$. Pick $x_{0} \in m_{2}(t) \cap \Omega$. Since $x_{0}$ is at the same time a local minimum and a global maximum for $h(t, \cdot)$, we can find an open subset $V$ of $\Omega$ such that $h(t, x)=u_{h}(t)$ for all $x \in V$. If we choose $n, k \in \mathbf{N}$ for which $B_{n, k} \subset V$ we easily get $\{0\}=h\left(t, B_{n, k} \cap \Omega\right)-u_{h}(t)$. Therefore, it follows that

$$
m_{2}^{-}(\Omega) \subseteq \bigcup_{(n, k) \in \mathbf{N} \times \mathbf{N}}\left\{t \in T_{2}:\{0\}=h\left(t, B_{n, k} \cap \Omega\right)-u_{h}(t)\right\}
$$

Since the reverse inclusion comes by the same argument, then (8) is proved. Finally, we claim that the multi-function $m_{3}$ is lower semicontinuous. To prove this latter assertion, fix an open subset $\Omega$ of $A$. We have to show that $m_{3}^{-}(\Omega)$ is open. Let $t_{0} \in m_{3}^{-}(\Omega)$ and $x_{0} \in m_{3}\left(t_{0}\right) \cap \Omega$. Therefore, $l_{h}\left(t_{0}\right)<h\left(t_{0}, x_{0}\right)<u_{h}\left(t_{0}\right)$ and $x_{0}$ is a local minimum for $h\left(t_{0}, \cdot\right)$. As $h$ is continuous, $l_{h}$ is upper semicontinuous and $u_{h}$ is lower semi-continuous, we can find an open 
neighborhood $V_{1}$ of $t_{0}$ and an open neighborhood $W_{1} \subseteq \Omega$ of $x_{0}$ in $A$ such that $l_{h}(t)<h(t, x)<u_{h}(t)$ for every $(t, x) \in V_{1} \times W_{1}$. Also, we can find an open neighborhood $W_{2}$ of $x_{0}$, with $W_{2} \subseteq W_{1}$, such that $h\left(t_{0}, x\right) \geq h\left(t_{0}, x_{0}\right)$ for every $x \in W_{2}$. Without loss of generality, we can suppose that $W_{2}$ is an interval. Now, suppose $x_{0} \in \operatorname{int}(A)$. Then, by condition (6), there are $x_{1}, x_{2} \in W_{2}$ satisfying $x_{1}<x_{0}<x_{2}, h\left(t_{0}, x_{1}\right)>h\left(t_{0}, x_{0}\right)$ and $h\left(t_{0}, x_{2}\right)>h\left(t_{0}, x_{0}\right)$. By the continuity of $h$, there exists an open neighborhood $V_{2}$ of $t_{0}$ with $V_{2} \subseteq V_{1}$ such that $h\left(t, x_{1}\right)>h\left(t, x_{0}\right)$ and $h\left(t, x_{2}\right)>h\left(t, x_{0}\right)$ for all $t \in V_{2}$. Consequently, if $t \in V_{2}$, the function $h(t, \cdot)$ has a local minimum $x_{t}$ lying in $] x_{1}, x_{2}\left[\subseteq W_{2} \subseteq W_{1} \subseteq \Omega\right.$. Analogously, if $x_{0}=\inf A$ or $x_{0}=\sup A$ we can argue in a similar manner to achieve the same conclusion. Consequently, $x_{t} \in m_{3}(t) \cap \Omega$ for every $t \in V_{2}$ and thus $m_{3}^{-}(\Omega)$ is open. In particular, the multi-function $m_{3}$ is measurable. Therefore, taking into account (7) and [8, Proposition 2.3], we have that the multi-function $m_{h}$ is measurable as well. By the same argument it can be proved that the multi-function $M_{h}$ is measurable.

By Lemma 2.2 the following result follows easily.

Lemma 2.3. Let $T$ and $A$ be as in Lemma 2.2 and let $\mu$ be a complete measure on $T$. Assume that $h: T \times A \rightarrow \mathbf{R}$ is measurable in first variable and continuous in the second one. Moreover, assume that (6) holds for almost all $t \in T$.

Then, the multi-functions $m_{h}, M_{h}$ are measurable.

Proof. Let $T_{0} \subseteq T$ such that $\mu\left(T_{0}\right)=0$ and (6) holds for all $t \in T_{1}:=T \backslash T_{0}$. By the Scorza-Dragoni theorem, we get the existence of a sequence of pairwise disjoint measurable sets $\left\{K_{n}\right\}$ in $T$ such that

$$
\mu\left(T_{1} \backslash \cup_{n \in \mathbf{N}} K_{n}\right)=0
$$

and the restriction of $h$ to each set $K_{n} \times A$ is continuous. By Lemma 2.2, the multi-functions $m_{h}$ and $M_{h}$ are measurable on each $K_{n}$. Since

$$
\mu\left(T_{0} \cup\left(T_{1} \backslash \cup_{n \in \mathbf{N}} K_{n}\right)\right)=0
$$

it follows that, the multi-functions $m_{h}$ and $M_{h}$ are measurable on $T$.

a 
Finally, we prove the following result.

Lemma 2.4. Let $T$ and $A$ be as in Lemma 2.2. Assume that $h: T \times A \rightarrow \mathbf{R}$ is measurable in first variable and continuous in the second one, and that $h$ satisfies condition (6). Moreover, assume that $m_{h}(t)$ and $M_{h}(t)$ are closed for all $t \in T$.

Then, there exists a multi-function $Y: T \rightarrow 2^{A}$ such that:

(i) the graph $\operatorname{Gr}(Y)$ of $Y$ is $\mathcal{T} \otimes \mathcal{B}(A)$-measurable;

(ii) for each measurable $J \subseteq \mathbf{R}$, and each $\mathcal{T} \otimes \mathcal{B}(J)$-measurable function $g: T \times J \rightarrow \mathbf{R}$ satisfying

$$
g(t, x) \in h(t, A) \quad \text { for all }(t, x) \in T \times J,
$$

the multi-function $H_{g}: T \times J \rightarrow 2^{A}$, defined by putting

$$
H(t, x):=h_{t}^{-1}(g(t, x)) \cap Y(t),
$$

is $\mathcal{T} \otimes \mathcal{B}(J)$-measurable and with closed values.

Proof. Let $J \subseteq \mathbf{R}$ be a measurable set, and let $g: T \times J \rightarrow \mathbf{R}$ be any $\mathcal{T} \otimes \mathcal{B}(J)$-measurable function satisfying (9). By [11, Theorem 2.4], if for $t \in T$ we take

$$
\begin{aligned}
Y(t)=\left[h_{t}^{-1}\left(l_{h}(t)\right) \cap\left(A \backslash M_{h}(t)\right)\right] \cup \\
{\left[h_{t}^{-1}\left(u_{h}(t)\right) \cap\left(A \backslash m_{h}(t)\right)\right] \cup\left[A \backslash\left(m_{h}(t) \cup M_{h}(t)\right)\right], }
\end{aligned}
$$

one has that $H(t, x)$ is nonempty and closed for all $(t, x) \in T \times J$. By Corollary 1 and Theorem 3.5 of $[8]$ we have that the graph of both the multi-functions $m_{h}$ and $M_{h}$ is $\mathcal{T} \otimes \mathcal{B}(A)$-measurable. Now, observe that one has

$$
\begin{aligned}
\operatorname{Gr}(Y)= & {\left[\left(h(\cdot, \cdot)-l_{h}(\cdot)\right)^{-1}(0) \cap\left(A \times T \backslash \operatorname{Gr}\left(M_{h}\right)\right)\right] } \\
& \cup\left[\left(h(\cdot, \cdot)-u_{h}(\cdot)\right)^{-1}(0) \cap\left(A \times T \backslash \operatorname{Gr}\left(m_{h}\right)\right)\right] \\
& \cup\left[A \times T \backslash\left(\operatorname{Gr}\left(m_{h}\right) \cup \operatorname{Gr}\left(M_{h}\right)\right)\right] .
\end{aligned}
$$

Consequently, $\operatorname{Gr}(Y)$ is $\mathcal{T} \otimes \mathcal{B}(A)$-measurable. Again by [8, Theorem $3.5]$, to achieve the conclusion it is enough to prove that $\operatorname{Gr}(H)$ is 
$\mathcal{T} \otimes \mathcal{B}(J) \otimes \mathcal{B}(A)$-measurable. This latter fact comes out from the following equality

$$
\operatorname{Gr}(H)=\{(t, x, y) \in T \times J \times A: h(t, y)=g(t, x) \text { and } y \in Y(t)\} .
$$

This ends the proof.

Proof of Theorem 1.1. Once the preliminary results have been established, the proof of Theorem 1.1 essentially follows the one of [1, Theorem 1.1], to which we will often refer in the sequel. Without loss of generality, we can assume that (3), (4), (5) and also assumption (ii) hold for all $t \in I$. Moreover, we can assume that $j<+\infty$ and that $h(t, \cdot)$ is continuous for all $t \in I$.

As shown in the proof of Theorem 1 of [1], the functions $v(t)$ and $z(t)$ are measurable in $I$. Let $l: I \rightarrow \mathbf{R}$ be any measurable function such that

$$
v(t) \leq l(t) \leq z(t) \text { for all } t \in I,
$$

and let $\hat{f}: I \times J \rightarrow \mathbf{R}$ be defined by

$$
\hat{f}(t, x)= \begin{cases}f^{*}(t, x) & \text { if } x \notin E_{2} \\ l(t) & \text { if } x \in E_{2} .\end{cases}
$$

As in [1], it can be checked that for each $t \in I$ one has

$$
\{x \in J: \hat{f}(t, \cdot) \text { is discontinuous at } x\} \subseteq E_{2},
$$

the function $\hat{f}$ is $\mathcal{L}(I) \otimes \mathcal{B}(J)$-measurable and one has

$$
v(t) \leq \hat{f}(t, x) \leq z(t) \quad \text { for all }(t, x) \in I \times J .
$$

For each $t \in I$, let $Y(t) \subseteq A$ be defined as in (10). From now on, for simplicity, we put $Y_{t}:=Y(t)$. By assumption (ii) and by [11, Theorem 2.4], one has that for each $t \in I$ the function

$$
\left.h_{t}\right|_{Y_{t}}: Y_{t} \longrightarrow h_{t}(A)
$$

is open and $h_{t}\left(Y_{t}\right)=h_{t}(A)$. It follows that for each $t \in I$ the multifunction $T_{t}: h_{t}(A) \rightarrow 2^{Y_{t}}$ defined by

$$
T_{t}(s)=h_{t}^{-1}(s) \cap Y_{t}
$$


is lower semi-continuous in $h_{t}(A)$ with nonempty values. Let $G$ : $I \times J \rightarrow 2^{A}$ be defined by

$$
G(t, x)=T_{t}(\hat{f}(t, x))=h_{t}^{-1}(\hat{f}(t, x)) \cap Y_{t}
$$

( $G$ is well defined by (5) and (12)). By Lemma 2.4, the multi-function $G$ is $\mathcal{L}(I) \otimes \mathcal{B}(J)$-measurable, with closed values and, by (11), for all $t \in I$ one has

$$
\{x \in J: G(t, \cdot) \text { is not lower semi-continuous at } x\} \subseteq E_{2} .
$$

Put

$$
E_{3}=\lambda\left\{\frac{p}{2^{m}}: p, m \in \mathbf{N}, p \leq 2^{m}\right\} .
$$

By [1, Lemma 8], there exists a function $k: I \times J \rightarrow \mathbf{R}$ such that

$$
k(t, x) \in G(t, x) \text { for all }(t, x) \in I \times J .
$$

Moreover, for a.a. $t \in I$, one has

$$
\{x \in J: k(t, \cdot) \text { is discontinuous at } x\} \subseteq E_{2} \cup E_{3}
$$

and for each $x \in J \backslash\left(E_{2} \cup E_{3}\right)$ the function $k(\cdot, x)$ is measurable. For each $t \in I$, let us put

$$
\alpha(t):=\inf h_{t}^{-1}([v(t), z(t)]) .
$$

By the continuity of $h_{t}$, taking into account (5) and (12), we get

$$
k(t, x) \in h_{t}^{-1}(\hat{f}(t, x)) \quad \text { for all }(t, x) \in I \times J
$$

and also

$$
0<\alpha(t) \leq k(t, x) \leq \beta(t) \text { for all }(t, x) \in I \times J .
$$

Let $T_{1} \subseteq I$ be such that $m\left(T_{1}\right)=0$ and (13) holds for all $t \in I \backslash T_{1}$. Define $\psi: I \times \mathbf{R} \rightarrow \mathbf{R}$ by putting

$$
\psi(t, x)= \begin{cases}k(t, x) & \text { if }(t, x) \in\left(I \backslash T_{1}\right) \times\left(J \backslash E_{2}\right) \\ \beta(t) & \text { otherwise. }\end{cases}
$$


Then, for each $t \in I \backslash T_{1}$, one has

$$
\{x \in \mathbf{R}: \psi(t, \cdot) \text { is discontinuous at } x\} \subseteq E_{2} \cup E_{3} .
$$

Let $P^{\prime}:=(\lambda(\mathbf{Q} \cap I)) \backslash E_{3}$ (where $\mathbf{Q}$ denotes the set of rational real numbers), and let $P^{\prime \prime}$ be any countable dense subset of $\mathbf{R} \backslash J$. Then the set $P^{*}:=P^{\prime} \cup P^{\prime \prime}$ is countable and dense in $\mathbf{R}$, and by the above construction the function $\psi(\cdot, x)$ is measurable for all $x \in P^{*}$.

Since the assumptions of Proposition 2 of [2] are fulfilled, the multifunction $F: I \times \mathbf{R} \rightarrow \mathbf{R}$ defined by

$$
F(t, x):=\bigcap_{m \in \mathbf{N}} \overline{\operatorname{co}}\left(\bigcup_{\substack{y \in P^{*} \\|y-x| \leq(1 / m)}}\{\psi(t, y)\}\right)
$$

satisfies the conclusion of the same Proposition (note that, since $\overline{\mathrm{co}}(\bar{A})=\overline{\mathrm{co}}(A)$ for $A \subseteq \mathbf{R}^{n}$, we have removed the closure of the union in the definition of $F$ given in Proposition 2 of [2]). Moreover,

$$
F(t, x) \subseteq[\alpha(t), \beta(t)] \text { for all }(t, x) \in I \times \mathbf{R} .
$$

Applying [1, Theorem 1] exactly in the same way as in $[\mathbf{1}]$, we get the existence of a function $\hat{u} \in L^{s}(I)$ and a set $T_{2} \subseteq I$, with $m\left(T_{2}\right)=0$, such that

$$
\hat{u}(t) \in F\left(t, \int_{I} g(t, z) \hat{u}(z) d z\right) \text { for all } t \in I \backslash T_{2} .
$$

At this point, we can argue exactly as in proof of Theorem 1 of $[\mathbf{1}]$ (the set $\lambda H$ being replaced by $E_{3}$ ) to deduce that $\hat{u}$ satisfies equation (2).

Example. We present an example of application of Theorem 1.1 where the nonlinearity $f$ is discontinuous at each point with respect to the second variable. Let $s \in] 1,+\infty[, \lambda, m, \gamma>0$ and $\sigma \in \mathbf{R}$ with $\sigma<1-(1 / s)$. Let $\alpha \in C([0,1] \times[0, \lambda])$ positive and satisfying

$$
\left\|\max _{x \in[0, \lambda]}(\alpha(\cdot, x))^{1 / m}\right\|_{L^{s}([0,1])} \leq \frac{\lambda}{2}\left(\frac{s(1-\sigma)-1}{s-1}\right)
$$


and put $f(t, x)=\alpha(t, x)\left(1+\chi_{\mathbf{Q}}(x)\right)$ for all $(t, x) \in[0,1] \times[0, \lambda]$, where $\chi_{\mathbf{Q}}(x)=1$ if $x \in \mathbf{Q}$ and $\chi_{\mathbf{Q}}(x)=0$ if $x \in \mathbf{R} \backslash \mathbf{Q}$ is the characteristic function of the set of the rational number $\mathbf{Q}$.

Finally, put

$$
h(t, y)=|y|^{m} e^{t y}
$$

for all $(t, y) \in[0,1] \times[0,+\infty]$ and

$$
g(t, z)=\frac{(1+t+z)^{\gamma}}{z^{\sigma}}
$$

if $(t, z) \in[0,1] \times] 0,1]$ (and arbitrarily defined in $[0,1] \times\{0\}$ ).

Then, Theorem 1.1 applies choosing $j=s^{\prime}, f^{*}=\alpha, P=$ $E_{1}=\mathbf{Q}, E_{2}=\varnothing, \phi_{1}(z)=\gamma, \phi_{0}(z)=\gamma 3^{\gamma} z^{-\sigma}$ and $\beta(t)=$ $\max _{x \in[0, \lambda]}(2 \alpha(t, x))^{1 / m}$.

Acknowledgments. The authors wish to thank the anonymous referees for their valuable suggestions and comments.

\section{REFERENCES}

1. G. Anello and P. Cubiotti, Non-autonomous implicit integral equations with discontinuous right-hand side, Comment. Math. Univ. Carolin. 45 (2004), 417-429.

2. F. Cammaroto and P. Cubiotti, Implicit integral equations with discontinuous right-hand side, Comment. Math. Univ. Carolin. 38 (1997), 241-246.

3. - Vector integral equations with discontinuous right-hand side, Comment. Math. Univ. Carolin. 40 (1999), 483-490.

4. P. Candito, Implicit integral equations with discontinuous nonlinearities, J. Integral Equations Appl. 14 (2002), 1-10.

5. C. Castaing and M. Valadier, Convex analysis and measurable multifunctions, Springer-Verlag, Berlin, 1977.

6. P. Cubiotti, Non-autonomous vector integral equations with discontinuous right-hand side, Comment. Math. Univ. Carolin. 42 (2001), 319-329.

7. M. Feckăn, Nonnegative solutions of nonlinear integral equations, Comment. Math. Univ. Carolin. 36 (1995), 615-627.

8. C.J. Himmelberg, Measurable relations, Fund. Math. 87 (1975), 53-72.

9. B. Kunze, On a special class of nonlinear integral equations J. Integral Equations Appl. 7 (1995), 329-350.

10. O. Naselli Ricceri and B. Ricceri, An existence theorem for inclusions of the type $\Psi(u)(t) \in F(t, \Phi(u)(t))$ and application to a multivalued boundary value problem, Appl. Anal. 38 (1990), 259-270. 
A NOTE ON NON-AUTONOMOUS IMPLICIT INTEGRAL EQUATIONS 403

11. B. Ricceri, Sur la semi-continuitè infèrieure de certaines multifonctions, C.R. Acad. Sci. Paris 294 (1982), 265-267.

Department of Mathematics, University of Messina, Contrada Papardo, Salita Sperone 31, 98166 Messina, Italy

Email address: anello@dipmat.unime.it

Email address: cubiotti@dipmat.unime.it 